PROCEEDINGS OF THE

AMERICAN MATHEMATICAL SOCIETY

Volume 125, Number 7, July 1997, Pages 2007-2011

S 0002-9939(97)03797-0

\title{
FORMULAE AND CONTINUITY FOR THE INDEX OF SUBFACTORS
}

\author{
SERGEY DOROFEEV AND KLAUS THOMSEN \\ (Communicated by Palle E. T. Jorgensen)
}

\begin{abstract}
Let $N \subset M$ be an inclusion of $I I_{1}$-factors, $\tau$ the trace state of $M$, and $\mathcal{P}(M), \mathcal{P}(N)$ the set of projections in $M$ and $N$, respectively. We prove that the Jones index for the inclusion is

$$
\begin{aligned}
{[M: N] } & =\sup _{e \in \mathcal{P}(M) \backslash\{0\}} \inf _{p \in \mathcal{P}(N) \backslash\{0\}} \frac{\tau(p)}{\tau(e p)} \\
& =\sup _{e \in \mathcal{P}(M) \backslash\{0\}} \text { inf }\left\{\frac{\tau(p)}{\tau(e p)}: p \in \mathcal{P}(N), e \preceq p\right\} .
\end{aligned}
$$

This formula is exploited to obtain continuity results for the index. In particular, we obtain a formula for the index which expresses $[M: N]$ in terms of the positions of $N_{i}$ and $M_{j}, i, j \in \mathbb{N}$, in $M$, when $N_{1} \subset N_{2} \subset N_{3} \subset \cdots$ and $M_{1} \subset M_{2} \subset M_{3} \subset \cdots$ are finite-dimensional $C^{*}$-subalgebras with dense union in $N$ and $M$, respectively.
\end{abstract}

It has been shown by Pimsner and Popa in $[\mathrm{PP}]$ that the index $[M: N]$ of an inclusion $N \subset M$ of $I I_{1}$ factors is the reciprocal of

$$
\max \left\{\lambda \in[0,1]: E(x) \geq \lambda x, x \in M^{+}\right\}
$$

where $E: M \rightarrow N$ is the trace-invariant conditional expectation. This formula is of crucial importance in the sub-factor theory, and it is the basis for most generalizations of the Jones index to other cases. It can also be used, in the setting of ergodic theory, to define the index of a sub- $\sigma$-algebra $\mathcal{B}_{0}$ of measurable subsets in a measure space $(X, \mathcal{B}, \mu)$ with $\mu(X)=1$. In this setup it served as a main tool in $[\mathrm{DT}]$, where it was shown that

$$
\begin{gathered}
\max \left\{\lambda \in[0,1]: E_{\mathcal{B}_{0}}(f) \geq \lambda f, f \in L^{\infty}(X, \mathcal{B}, \mu)^{+}\right\} \\
=\max \left\{\lambda \in[0,1]: \forall A \in \mathcal{B} \text { there is a } C \in \mathcal{B}_{0}\right. \text { such that } \\
\qquad(A \backslash C)=0 \text { and } \lambda \mu(C) \leq \mu(A)\} .
\end{gathered}
$$

The following result shows that this formula also holds in the non-commutative case of $I I_{1}$-factors.

Received by the editors January 16, 1996.

1991 Mathematics Subject Classification. Primary 46L37.

(C)1997 American Mathematical Society 
Theorem 1. Let $M$ be a $I I_{1}$-factor and $N \subset M$ a subfactor and $\mathcal{P}(N), \mathcal{P}(M)$ the projection lattices in $N$ and $M$, respectively. Then

$$
\begin{aligned}
& {[M: N]^{-1}} \\
& =\max \left\{\lambda \in[0,1]: \forall e \in \mathcal{P}(M) \exists p \in \mathcal{P}(N) \text { such that } e \preceq p \text { and } \lambda p \leq p E_{N}(e)\right\} \\
& =\max \{\lambda \in[0,1]: \forall e \in \mathcal{P}(M) \exists p \in \mathcal{P}(N) \text { such that } e \preceq p \text { and } \lambda \tau(p) \leq \tau(e p)\} \\
& =\max \{\lambda \in[0,1]: \forall e \in \mathcal{P}(M) \backslash\{0\} \exists p \in \mathcal{P}(N) \backslash\{0\} \text { such that } \lambda \tau(p) \leq \tau(e p)\} .
\end{aligned}
$$

Proof. Set $A=\{\lambda \in[0,1]: \forall e \in \mathcal{P}(M) \exists p \in \mathcal{P}(N)$ such that $e \preceq p$ and $\lambda p \leq$ $\left.p E_{N}(e)\right\}, B=\{\lambda \in[0,1]: \forall e \in \mathcal{P}(M) \exists p \in \mathcal{P}(N)$ such that $e \preceq p$ and $\lambda \tau(p) \leq$ $\tau(e p)\}$ and $C=\{\lambda \in[0,1]: \forall e \in \mathcal{P}(M) \backslash\{0\} \exists p \in \mathcal{P}(N) \backslash\{0\}$ such that $\lambda \tau(p) \leq$ $\tau(e p)\}$. To handle the least interesting case first, assume that $[M: N]=\infty$. In this case we see from $[\mathrm{PP}]$, Theorem 2.2, that for all $\epsilon>0$ there is a non-zero projection $e \in M$ such that $\left\|E_{N}(e)\right\|<\epsilon$. From this it follows straightforwardly that $A=B=$ $C=\{0\}$. Now we assume that $[M: N]^{-1}>0$. If $\lambda \in A$ and $e \in \mathcal{P}(M)$ we have a $p \in \mathcal{P}(N)$ such that $e \preceq p$ and $\lambda p \leq p E_{N}(e)$. Then $\lambda \tau(p) \leq \tau\left(p E_{N}(e)\right)=\tau(e p)$. Thus $A \subset B$. It is trivial that $B \subset C$. Let $\mu \in C$. By $[\mathrm{J}]$ and $[\mathrm{PP}]$ there is a projection $e \in M$ such that $E_{N}(e)=[M: N]^{-1} 1$. In particular, $e \neq 0$ and we have a non-zero projection $p \in N$ such that $\mu \tau(p) \leq \tau(e p)=\tau\left(E_{N}(e) p\right)=[M: N]^{-1} \tau(p)$, showing that $\mu \leq[M: N]^{-1}$. Thus sup $A \leq \sup B \leq \sup C \leq[M: N]^{-1}$ and the proof can be completed by showing that $[M: N]^{-1} \in A$.

Set $\lambda=[M: N]^{-1}$. By Proposition 2.1 of $[\mathrm{PP}]$ we have that $E_{N}(x) \geq \lambda x$ for all $x \in M_{+}$. The proof that $\lambda \in A$ is based on this and the following simple observation:

When $a \in M_{+}, p \in \mathcal{P}(M) \backslash\{0\}, s, t \in[0, \infty[$

such $s<t$ and $t p \leq a$, then the spectral projection of $a$ corresponding to the interval $[s, \infty[$ is non-zero.

Consider $e \in \mathcal{P}(M) \backslash\{0\}$ and fix $n \in \mathbb{N}$. Since $\left(\lambda+\frac{1}{n}\right) e \leq E_{N}(e)+\frac{1}{n} 1$ we see from (1) that there is a non-zero projection $q$ in the abelian von Neumann algebra $\mathcal{A}$ generated by $E_{N}(e)$ and 1 such that $q\left(E_{N}(e)+\frac{1}{n} 1\right) \geq \lambda q$. Let $\left\{q_{i}\right\}$ be a maximal family of non-zero mutually orthogonal projections in $\mathcal{A}$ such that $q_{i}\left(E_{N}(e)+\frac{1}{n} 1\right) \geq$ $\lambda q_{i}$ for all $i$. Set $f_{n}=\sum_{i} q_{i} \in \mathcal{A}$ and note that $f_{n}\left(E_{N}(e)+\frac{1}{n} 1\right) \geq \lambda f_{n}$. Note that

$$
\begin{aligned}
\left(\lambda+\frac{1}{n}\right) e \wedge\left(1-f_{n}\right) & \leq \lambda\left(1-f_{n}\right) e\left(1-f_{n}\right)+\frac{1}{n}\left(1-f_{n}\right) \\
& \leq\left(1-f_{n}\right)\left(E_{N}(e)+\frac{1}{n} 1\right)\left(1-f_{n}\right)
\end{aligned}
$$

which shows that $e \wedge\left(1-f_{n}\right)=0$, since otherwise (1) would give us a non-zero projection $q$ orthogonal to $f_{n}$ such that $q\left(E_{N}(e)+\frac{1}{n} 1\right) \geq \lambda q$, contradicting the maximality of $\left\{q_{i}\right\}$. Now the parallelogram rule shows that $e \sim f_{n}-(1-e) \wedge f_{n} \leq f_{n}$, so that $\tau(e) \leq \tau\left(f_{n}\right)$. Set $p_{n}=\bigvee_{i=n}^{\infty} f_{i}$ and observe that $p_{n}\left(E_{N}(e)+\frac{1}{n} 1\right) \geq \lambda p_{n}$ and that $\tau\left(p_{n}\right) \geq \tau\left(f_{n}\right) \geq \tau(e)$. Since $\left\{p_{n}\right\}$ is a decreasing sequence of projections the strong limit $p=\lim _{n \rightarrow \infty} p_{n}$ is a projection. As $\tau(p)=\lim _{n} \tau\left(p_{n}\right) \geq \tau(e)$ we have that $e \preceq p$. Since $p_{n}\left(E_{N}(e)+\frac{1}{n} 1\right) \geq \lambda p_{n}$ for all $n$ we find that $p E_{N}(e) \geq \lambda p$.

By taking the reciprocal value of the last two expressions of Theorem 1 we get the formulae of the abstract for the index. 
One virtue of the alternative expressions for the index obtained in Theorem 1 is that they have nice continuity properties. To illustrate this we prove two results where this is exploited. Consider a type $I I_{1}$ factor $B$ with trace state $\tau$ and two von Neumann subalgebras, $N$ and $M$, of $B$. This setting will be fixed throughout the rest of this note. Set

$$
\begin{gathered}
\lambda(M, N)=\sup \{\lambda \in[0,1]: \forall e \in \mathcal{P}(M) \exists p \in \mathcal{P}(N) \text { such that } \\
\tau(e) \leq \tau(p) \text { and } \lambda \tau(p) \leq \tau(e p)\} .
\end{gathered}
$$

By Theorem 1 we have that $\lambda(M, N)^{-1}=[M: N]$ when $M=B$ and $N$ is a factor.

Theorem 2. Assume that $\overline{\{\tau(e): e \in \mathcal{P}(M)\}}=\overline{\{\tau(e): e \in \mathcal{P}(N)\}}=[0,1]$. Let $M_{1} \subset M_{2} \subset M_{3} \subset \cdots$ and $N_{1} \subset N_{2} \subset N_{3} \subset \cdots$ be sequences of finite dimensional $C^{*}$-subalgebras of $M$ and $N$ such that $\bigcup_{k} M_{k}$ and $\bigcup_{k} N_{k}$ are strongly dense in $M$ and $N$, respectively. It follows that

$$
\lambda(M, N)=\lim _{k \rightarrow \infty} \lim _{l \rightarrow \infty} \lambda\left(M_{k}, N_{l}\right) .
$$

For the proof we shall need the following lemma.

Lemma 3. Let $A_{1} \subset A_{2} \subset A_{3} \subset \cdots$ be finite dimensional unital $C^{*}$-subalgebras of $B$ and let $A$ be the strong closure of $\bigcup_{k} A_{k}$. Assume that $\overline{\{\tau(e): e \in \mathcal{P}(A)\}}=[0,1]$.

Let $p \in \mathcal{P}(A), \epsilon>0$. There are then a $k \in \mathbb{N}$ and a projection $q \in A_{k}$ such that $\|p-q\|_{2}<\epsilon$ and $\tau(p) \leq \tau(q)$.

Proof. Let $0<\delta$ such that $\delta+\sqrt{3 \delta}<\epsilon$. It is well known that there are a $k \in \mathbb{N}$ and a projection $q_{0} \in A_{k}$ such that $\left\|p-q_{0}\right\|_{2}<\delta$. By increasing $k$ we may assume that $\left\{\tau(e): e \in \mathcal{P}\left(A_{k}\right)\right\}$ is $\delta$-dense in $[0,1]$. Let $r_{i}, i=1,2, \cdots, m$, be the minimal nonzero central projections in $A_{k}$. We can choose a projection $q \in A_{k}$ such that $|\tau(q)-(\tau(p)+\delta)| \leq \delta$ and for each $i=1,2, \cdots, m, q r_{i} \leq q_{0} r_{i}$ or $q r_{i} \geq q_{0} r_{i}$. Then $\tau(q) \geq \tau(p)$ and

$$
\begin{aligned}
& \|q-p\|_{2} \leq \delta+\left\|q_{0}-q\right\|_{2}=\delta+\sqrt{\left|\tau\left(q_{0}-q\right)\right|} \leq \\
& \delta+\sqrt{\left|\tau\left(q_{0}\right)-\tau(p)\right|+|\tau(p)-\tau(q)|} \leq \delta+\sqrt{3 \delta}<\epsilon .
\end{aligned}
$$

Proof of Theorem 2. It is obvious that $\lambda\left(M_{k}, N_{l}\right)$ is increasing in $l$ for fixed $k$ and decreasing in $k$ for fixed $l$. We first prove that

$$
\lim _{l \rightarrow \infty} \lambda\left(M_{k}, N_{l}\right)=\lambda\left(M_{k}, N\right), k \in \mathbb{N}
$$

Since $\lambda\left(M_{k}, N_{l}\right) \leq \lambda\left(M_{k}, N\right)$ for all $l$, we have trivially that $\lim _{l \rightarrow \infty} \lambda\left(M_{k}, N_{l}\right) \leq$ $\lambda\left(M_{k}, N\right)$. Let $\epsilon>0$. By definition of $\lambda\left(M_{k}, N\right)$ there is for all $e \in \mathcal{P}\left(M_{k}\right) \backslash\{0\}$ a projection $p \in N$ such that $\tau(e) \leq \tau(p)$ and $\tau(e p)>\left(\lambda\left(M_{k}, N\right)-\epsilon\right) \tau(p)$. By the density of $\bigcup_{l} N_{l}$ in $N$ and Lemma 3 there are then an $i \in \mathbb{N}$ and a projection $p_{i} \in N_{i}$ such that $\tau(e) \leq \tau\left(p_{i}\right)$ and $\tau\left(e p_{i}\right)>\left(\lambda\left(M_{k}, N\right)-\epsilon\right) \tau\left(p_{i}\right)$. If $\delta<\min \left\{1, \epsilon \tau\left(p_{i}\right)\right\}$ and $e^{\prime} \in \mathcal{P}\left(M_{k}\right)$ such that $\left\|e-e^{\prime}\right\|<\delta$, then $e \sim e^{\prime}$ and hence $\tau(e)=\tau\left(e^{\prime}\right)$ for all $e^{\prime}$ in this neighbourhood of $e$. Since

$$
\tau\left(e^{\prime} p_{i}\right) \geq \tau\left(e p_{i}\right)-\delta \geq\left(\lambda\left(M_{k}, N\right)-\epsilon\right) \tau\left(p_{i}\right)-\delta \geq\left(\lambda\left(M_{k}, N\right)-2 \epsilon\right) \tau\left(p_{i}\right),
$$

we can use the compactness of the set of non-zero projections in $M_{k}$ to find $j \in \mathbb{N}$ such that $\forall e \in \mathcal{P}\left(M_{k}\right) \backslash\{0\}$ there is a $p \in \mathcal{P}\left(N_{j}\right)$ such that $\tau(e) \leq \tau(p)$ and 
$\tau(e p) \geq\left(\lambda\left(M_{k}, N\right)-2 \epsilon\right) \tau(p)$. It follows that $\lambda\left(M_{k}, N_{j}\right) \geq \lambda\left(M_{k}, N\right)-2 \epsilon$, proving (2). The next step is to prove that

$$
\lim _{k \rightarrow \infty} \lambda\left(M_{k}, N\right)=\lambda(M, N) .
$$

Since $\lambda\left(M_{k}, N\right) \geq \lambda(M, N)$ for all $k$ we obviously have that $\lim _{k \rightarrow \infty} \lambda\left(M_{k}, N\right) \geq$ $\lambda(M, N)$. Let $\epsilon>0$; then there exists an $e \in \mathcal{P}(M) \backslash\{0\}$ such that $\tau(e p)<$ $(\lambda(M, N)+\epsilon) \tau(p)$ for all $p \in \mathcal{P}(N)$ with $\tau(e) \leq \tau(p)$. By Lemma 3 there exist $k \in \mathbb{N}$ and a projection $e_{k} \in M_{k}$ such that $\left\|e-e_{k}\right\|_{2} \leq \tau(e) \epsilon$ and $\tau(e) \leq \tau\left(e_{k}\right)$. Thus, if $p \in \mathcal{P}(N)$ and $\tau(p) \geq \tau\left(e_{k}\right)$, we have

$$
\tau\left(e_{k} p\right) \leq \tau(e p)+\tau(e) \epsilon<(\lambda(M, N)+\epsilon) \tau(p)+\tau(e) \epsilon \leq(\lambda(M, N)+2 \epsilon) \tau(p),
$$

proving that $\lambda\left(M_{k}, N\right)<\lambda(M, N)+2 \epsilon$.

Note that it does not give anything interesting if we exchange the order in which the two limits are taken in Theorem 2. Indeed, the proof of (3) gives that $\lim _{k \rightarrow \infty} \lambda\left(M_{k}, N_{l}\right)=\lambda\left(M, N_{l}\right), l \in \mathbb{N}$. But $\lambda\left(M, N_{l}\right)=0$ since $M$ contains projections with arbitrarily small non-zero trace while inf $\left\{\tau(e): e \in \mathcal{P}\left(N_{l}\right) \backslash\{0\}\right\}>0$.

For each $\delta \in[0,1]$ set

$$
\begin{array}{r}
\lambda_{\delta}(M, N)=\sup \{t \in[0,1]: \forall e \in \mathcal{P}(M), \tau(e) \geq \delta \exists p \in \mathcal{P}(N) \\
\text { such that } \tau(e) \leq \tau(p) \text { and } \tau(e p) \geq t \tau(p)\} .
\end{array}
$$

Clearly, $\lim _{\delta \rightarrow 0} \lambda_{\delta}(M, N)=\lambda_{0}(M, N)=\lambda(M, N)$. We will use $\lambda_{\delta}(M, N)$ to investigate to what extent $\lambda(M, N)$ is continuous in $M, N$. By [MT], Theorem 4, we cannot expect any global continuity of $\lambda(M, N)$. However, $\lambda_{\delta}$ is always continuous when $\delta>0$. We define

$$
d_{0}(M, N)=\sup _{e \in \mathcal{P}(M)} \inf _{p \in \mathcal{P}(N)}\|e-p\|_{2},
$$

and

$$
D(M, N)=\max \left\{d_{0}(N, M), d_{0}(M, N)\right\} .
$$

$D$ is clearly a metric on the set of von Neumann subalgebras of $B$; in fact $D(M, N)$ is the Hausdorff distance between the projection lattices in $N$ and $M$. We say that a von Neumann subalgebra $N$ of $B$ is $\tau$-continuous when

$$
\{\tau(q): q \in \mathcal{P}(N), q \leq p\}=[0, \tau(p)]
$$

for all $p \in \mathcal{P}(N)$. By adopting the arguments from Lemma 3 we get the following

Lemma 4. Let $N_{1}, N_{2}$ be $\tau$-continuous von Neumann subalgebras of $B$. For any projection $e_{1} \in N_{1}$ and $\epsilon>0$ there is a projection $e_{2} \in N_{2}$ such that $\tau\left(e_{1}\right)=\tau\left(e_{2}\right)$ and $\left\|e_{1}-e_{2}\right\|_{2} \leq D\left(N_{1}, N_{2}\right)+\sqrt{D\left(N_{1}, N_{2}\right)}+\epsilon$.

It is straightforward to use Lemma 4 to prove

Lemma 5. Let $M_{1}, M_{2}, N_{1}, N_{2}$ be $\tau$-continuous von Neumann subalgebras of $B$. Then

$$
\begin{aligned}
& \left|\lambda_{\delta}\left(M_{1}, N_{1}\right)-\lambda_{\delta}\left(M_{2}, N_{2}\right)\right| \leq \\
& \quad \delta^{-\frac{1}{2}}\left(D\left(M_{1}, M_{2}\right)+\sqrt{D\left(M_{1}, M_{2}\right)}+D\left(N_{1}, N_{2}\right)+\sqrt{D\left(N_{1}, N_{2}\right)}\right) .
\end{aligned}
$$

Lemma 5 leads to the following improvement of Theorem 6 of [MT]. 
Theorem 6. Assume that $M_{1}, M_{2}, N_{1}, N_{2}$ are $I I_{1}$-factors contained in a common ambient II $I_{1}$-factor such that $N_{1} \subset M_{1}$ and $N_{2} \subset M_{2}$. It follows that

$$
\begin{aligned}
& \frac{\left|\left[M_{1}: N_{1}\right]^{-1}-\left[M_{2}: N_{2}\right]^{-1}\right|}{\max \left\{\left[M_{1}: N_{1}\right]^{\frac{1}{2}},\left[M_{2}: N_{2}\right]^{\frac{1}{2}}\right\}} \leq \\
& D\left(M_{1}, M_{2}\right)+\sqrt{D\left(M_{1}, M_{2}\right)}+D\left(N_{1}, N_{2}\right)+\sqrt{D\left(N_{1}, N_{2}\right)} .
\end{aligned}
$$

Proof. The inequality is trivial if $\left[M_{1}: N_{1}\right]$ or $\left[M_{2}: N_{2}\right]$ is $\infty$, so we may assume that $\max \left\{\left[M_{1}: N_{1}\right],\left[M_{2}: N_{2}\right]\right\}<\infty$. By Theorem 1, $\left[M_{i}: N_{i}\right]^{-1}=$ $\lambda\left(M_{i}, N_{i}\right), i \in\{1,2\}$. But there are projections (the Jones projections) $e_{i} \in M_{i}$ such that $E_{N_{i}}\left(e_{i}\right)=\lambda\left(M_{i}, N_{i}\right) 1$, so we see that $\lambda\left(M_{i}, N_{i}\right)=\lambda_{\delta}\left(M_{i}, N_{i}\right), i=1,2$, when $\delta=\min \left\{\lambda\left(M_{1}, N_{1}\right), \lambda\left(M_{2}, N_{2}\right)\right\}$. Apply Lemma 5 .

\section{REFERENCES}

[DT] S. Dorofeev, K. Thomsen, Factors and subfactors arising from inductive limits of interval algebras, Preprint, Aarhus, 1996.

[J] V. Jones, Index for subfactors, Invent. Math. 72 (1983), 1 - 25. MR 84d:46097

$[\mathrm{MT}]$ B. Mashhood, K.F. Taylor, On the continuity of the Index of Subfactors of a Finite Factor, J. Functional Analysis 76 (1988), 56-66. MR 89h:46090

[PP] M. Pimsner, S. Popa, Entropy and index for subfactors, Ann. Sci. Ecole. Norm. Sup. 19 (1986), 57-106. MR 87m:46120

Matematisk Institut, Ny Munkegade, 8000 Aarhus C, Denmark

E-mail address: dorofeev@mi.aau.dk

E-mail address: matkt@mi.aau.dk 